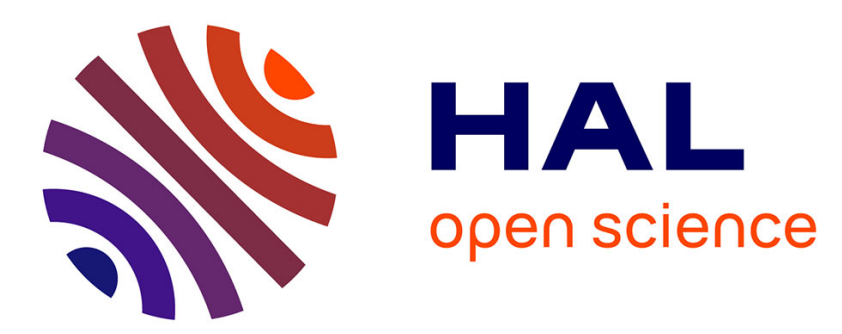

\title{
Oxydation du silicium par bombardement électronique. Influence de la pollution par le carbone
}

\author{
L. Pilorget, J.L. Chartier, R. Le Bihan
}

\section{To cite this version:}

L. Pilorget, J.L. Chartier, R. Le Bihan. Oxydation du silicium par bombardement électronique. Influence de la pollution par le carbone. Revue de Physique Appliquée, 1985, 20 (3), pp.151-155. 10.1051/rphysap:01985002003015100 . jpa-00245317

\section{HAL Id: jpa-00245317 https://hal.science/jpa-00245317}

Submitted on 1 Jan 1985

HAL is a multi-disciplinary open access archive for the deposit and dissemination of scientific research documents, whether they are published or not. The documents may come from teaching and research institutions in France or abroad, or from public or private research centers.
L'archive ouverte pluridisciplinaire HAL, est destinée au dépôt et à la diffusion de documents scientifiques de niveau recherche, publiés ou non, émanant des établissements d'enseignement et de recherche français ou étrangers, des laboratoires publics ou privés. 


\title{
Oxydation du silicium par bombardement électronique. Influence de la pollution par le carbone $\left(^{+}\right)$
}

\author{
L. Pilorget, J. L. Chartier et R. Le Bihan
}

Laboratoire de Physique des Surfaces (*), Institut de Physique, Université de Nantes, 44072 Nantes Cedex, France

(Reçu le 5 mai 1984, révisé le 13 novembre, accepté le 26 novembre 1984)

\begin{abstract}
Résumé. - L'oxydation du silicium monocristallin a été effectuée par bombardement électronique dans les trois conditions suivantes : 1) dans l'atmosphère résiduelle de l'enceinte à vide après isolation du groupe de pompage, 2) dans les mêmes conditions après introduction d'oxygène sec, 3) en présence d'un flux constant d'oxygène sec dans l'enceinte à vide. Les oxydes sont analysés par spectrométrie Auger en cours d'oxydation. Les oxydes obtenus sont comparés entre eux et à l'oxyde thermique. L'influence de l'atmosphère résiduelle est montrée.
\end{abstract}

\begin{abstract}
Silicon oxydation is realized by an electron beam in the three following conditions : 1) under the residual atmosphere in the vacuum chamber after closing the pumping connexion, 2) under the same conditions and introduction of dry $\left.\mathrm{O}_{2}, 3\right)$ under a constant flow of dry oxygen in the vacuum chamber. Silicon oxides are analysed by Auger spectrometry during the oxidation. The results obtained in these three cases are compared between them and with a thermal silicon oxide. The influence of residual gases on the results is shown.
\end{abstract}

\section{Introduction.}

Le développement de la microélectronique conduit à la réalisation de couches d'oxyde de silicium de plus en plus minces et, comme nous l'avons montré par ailleurs [1], il semble que l'homogénéité de ces couches soit liée aux premières phases de l'oxydation du silicium. Dans un premier temps, nous avons étudié l'oxydation sous bombardement électronique dans l'enceinte d'analyse. Ces études sont conduites dans le cadre du GCIS sur des substrats de Si (100) fournis par le LETI (fabrication Wacker).

\section{Partie expérimentale.}

L'oxydation a été réalisée sur du silicium d'orientation cristalline (100) dans les trois conditions suivantes :

- Sous ambiance résiduelle de l'enceinte ultravide, après isolation du groupe de pompage.

- Dans les mêmes conditions mais après introduction d'oxygène sous une pression partielle donnée.

- Sous flux constant d'oxygène. La pression partielle d'oxygène dans l'enceinte est le résultat de

$\left({ }^{+}\right)$Communication présentée aux Journées du G.C.I.S., Toulouse les 15 et 16 décembre 1983.

(*) U.A. CNRS no 788. l'équilibre entre le flux d'entrée d'oxygène et le pompage de l'enceinte.

La surface est bombardée par un faisceau d'électrons d'énergie 1 ou $3 \mathrm{keV}$ avec un courant de faisceau de $2,5 \times 10^{-7} \mathrm{~A}$, un diamètre de $50 \mu \mathrm{m}$ à $1 \mathrm{keV}$ et $30 \mu \mathrm{m}$ à $3 \mathrm{keV}$. Pour évaluer l'oxydation, des spectres Auger sont enregistrés à intervalles réguliers. Lorsque l'oxydation est terminée et après pompage de l'enceinte, un spectre de contrôle est effectué au même endroit ainsi qu'en un point situé en dehors de la zone précédemment bombardée pour vérifier que l'oxydation n'a lieu que sous le faisceau. L'analyse des gaz résiduels dans l'enceinte est effectuée à l'aide de l'« analyseur quadripolaire de gaz résiduel Miniquad" de chez C.V.C. permettant l'analyse des gaz de masse atomique comprise entre 2 et 100 U.M.A.

Les substrats de silicium sont préalablement décapés avec une solution à base d'acide fluorhydrique afin d'ôter la couche d'oxyde natif en surface. L'échantillon est ensuite rincé à l'eau désionisée, séché puis introduit dans l'enceinte ultra-vide. La seconde phase du nettoyage se fait sous vide, par décapage ionique aux ions argon : la surface est bombardée par le faisceau d'ions qui balaie la surface. Par spectrométrie Auger, on vérifie alors la propreté de la surface de l'échantillon. L'oxydation a lieu sous le faisceau électronique à la température ambiante. 


\section{Résultats. Discussion.}

Voyons successivement les différentes études effectuées :

3.1 OXYDATION PAR BOMBARDEMENT ÉLECTRONIQUE SOUS ATMOSPHÈRE RÉSIDUELLE. - Le relevé des pressions partielles des gaz s'effectue simultanément à l'oxydation, la figure 1 représente leur évolution au cours du temps. On remarquera une remontée importante des pressions de $\mathrm{CH}_{2}$ et de $\mathrm{CO}-\mathrm{N}_{2}$.

La figure 2 montre l'évolution des spectres Auger relevés régulièrement au cours de l'oxydation. Les phases caractéristiques sont les suivantes :

- Le spectre initial (a) montre le pic à $92 \mathrm{eV}$ caractéristique du silicium, il y a ensuite une déformation de ce pic dans sa partie haute énergie (spectre b), phénomène qui indique une saturation de la couche adsorbée et qui a été observé par ailleurs sur le silicium $[2,3]$.

- Apparition des pics à 80 et $84 \mathrm{eV}$, caractéristiques de l'oxydation du silicium (spectre c).

- La durée du bombardement électronique augmentant, seul le pic à $80 \mathrm{eV}$ subsiste, le pic à $92 \mathrm{eV}$ disparaît (spectres $d$ et e). Parallèlement l'énergie du pic oxygène est passée de $510 \mathrm{eV}$ sur le silicium à $507 \mathrm{eV}$ sur l'oxyde. Cette variation d'énergie est la même que celle observée pendant une oxydation thermique du silicium [1 et 4]. Quand on augmente la durée du bombardement, le spectre (e) persiste en

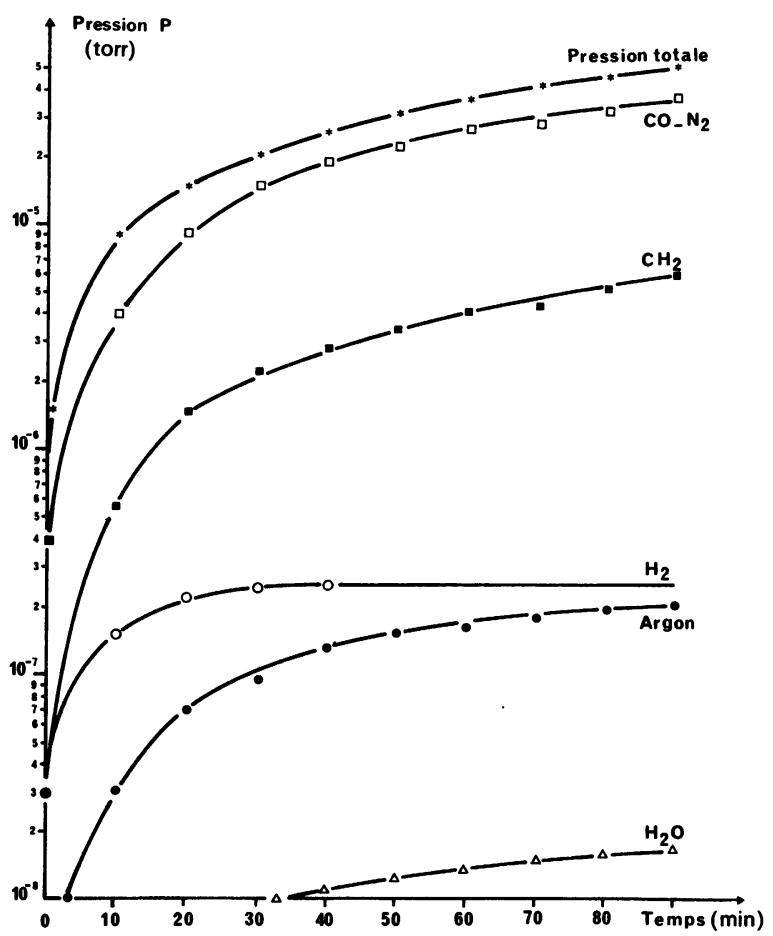

Fig. 1. - Evolution des pressions partielles dans l'enceinte après fermeture de la vanne et pendant le bombardement par le faisceau électronique.

[Partial pressures variation in the device after closing the vacuum gate.]

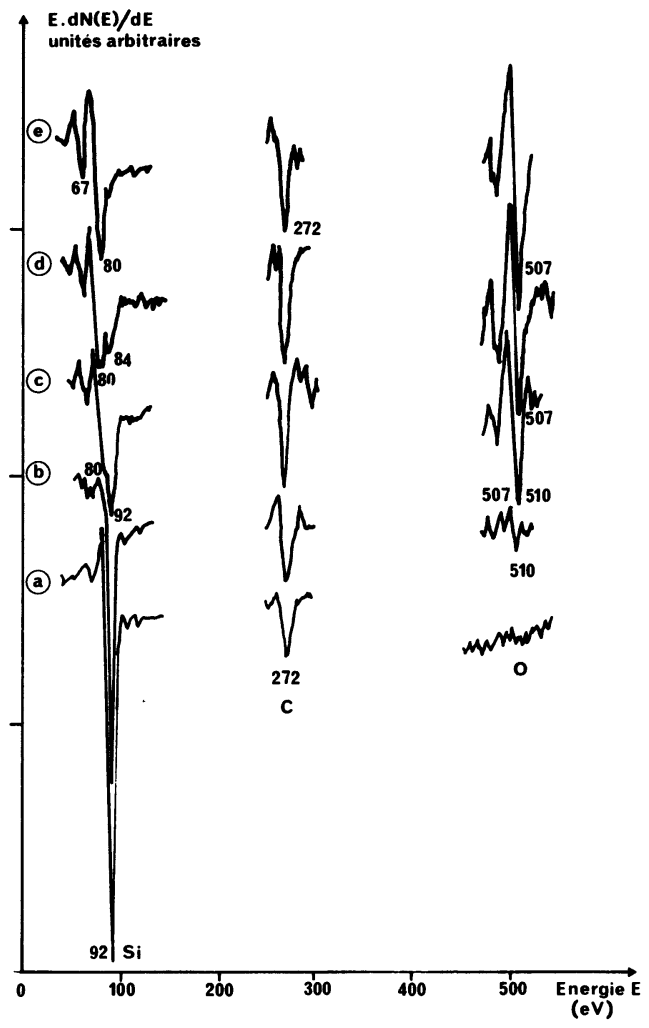

Fig. 2. - Evolution des spectres Auger du silicium à l'oxyde de silicium pendant l'oxydation sous faisceau électronique d'intensité $2,5 \times 10^{-7} \mathrm{~A}$ et d'énergie $1 \mathrm{keV}$.

[Auger spectra evolution when we oxide silicon under electron beam of $2.5 \times 10^{-7} \mathrm{~A}$ and $1 \mathrm{keV}$.]

conservant l'amplitude des signaux. On constate que les énergies des pics de l'oxyde sont décalés de $4 \mathrm{eV}$ par rapport à ceux obtenus sur un oxyde élaboré par croissance thermique et nettoyé par bombardement ionique [1] $(63,67$ et $80 \mathrm{eV}$ au lieu de 59, 63, $76 \mathrm{eV})$; par contre sur un oxyde thermique non nettoyé, on obtient les mêmes valeurs.

L'oxydation terminée, on fait un vide de quelque $10^{-9}$ torr dans l'enceinte et le spectre Auger relevé confirme le spectre (e). Sur la figure 3 , nous avons tracé la variation des amplitudes des différents signaux Auger caractéristiques de l'évolution de l'oxydation; l'amplitude des signaux étant normée par rapport au signal le plus important de même énergie observé au cours de l'expérience. Nous avons une décroissance du pic $92 \mathrm{eV}$ du silicium, une croissance du pic $80 \mathrm{eV}$ et de l'oxygène relatifs au silicium oxydé. Le spectre enregistré sur l'échantillon en dehors de l'impact électronique ne révèle pas d'oxydation sur la surface, seule la déformation du pic à $92 \mathrm{eV}$ permet de déterminer une couche d'oxygène adsorbée en surface.

Nous avons fait la même expérience en introduisant de l'argon sous une pression partielle de $2 \times 10^{-5}$ torr dans l'enceinte, les résultats obtenus sont les mêmes que ceux que nous venons de présenter. 


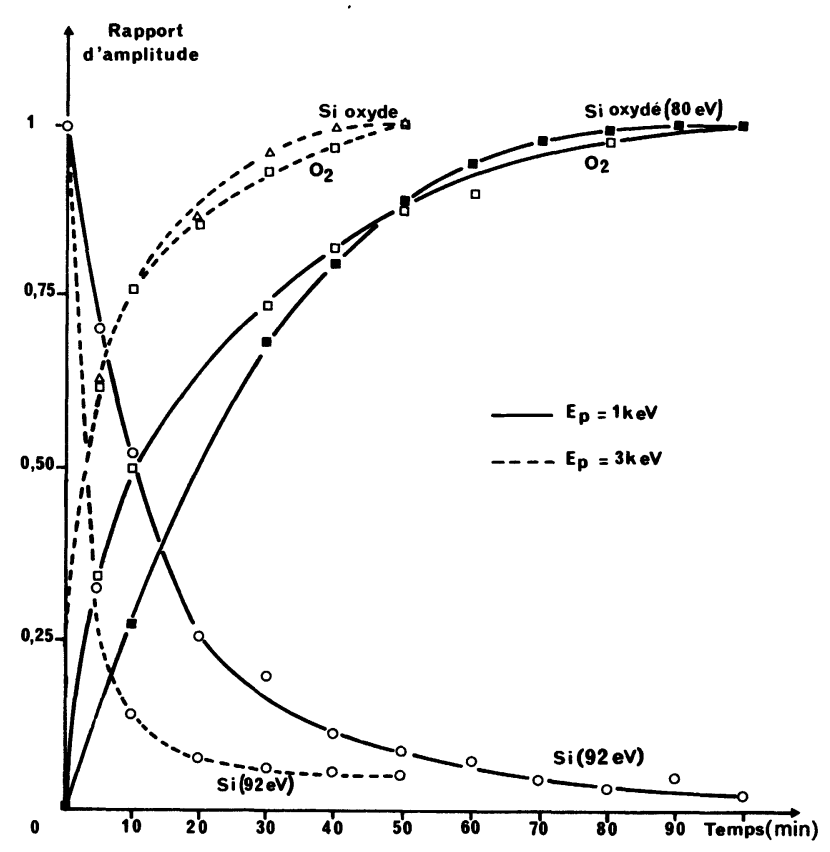

Fig. 3. - Evolution de l'amplitude des pics Auger au cours de l'oxydation pour un courant primaire $I_{\mathrm{p}}=2,5 \times 10^{-7} \mathrm{~A}$ et une énergie des électrons $E_{\mathrm{p}}=1 \mathrm{keV}$ ou $3 \mathrm{keV}$.

[Evolution of Auger peaks amplitude during the oxidation for a primary current $I_{\mathrm{p}}=2.5 \times 10^{-7} \mathrm{~A}$ and electrons energy $E_{\mathrm{p}}=1 \mathrm{keV}$ or $3 \mathrm{keV}$.]

L'oxydation est d'autant plus rapide que l'énergie primaire est plus importante, ainsi pour une énergie des électrons primaires de $3 \mathrm{keV}$ la vitesse d'oxydation est environ 3 fois plus grande que pour $1 \mathrm{keV}$ et la phase caractérisée par le pic Auger à $84 \mathrm{eV}$ n'apparaît pas.

3.2 OXYDATION PAR BOMBARDEMENT ÉLECTRONIQUE SOUS OXYGÈNE STATIQUE. - Outre la préparation indiquée précédemment pour les échantillons, nous avons ici fait subir un chauffage in situ à $600^{\circ} \mathrm{C}$ sous une pression totale d'environ $10^{-8}$ torr; après un nouveau décapage de la surface par bombardement aux ions argon, le contrôle par spectrométrie Auger ne fait apparaître aucune trace de contaminants, seuls apparaissent les pics du silicium pur à 74 et $92 \mathrm{eV}$. Après arrêt du pompage, on fait une introduction d'oxygène sec dans l'enceinte sous une pression de $5 \times 10^{-5}$ torr. Le spectre relevé à l'analyseur quadrupolaire indique une forte pression partielle du composé $\mathrm{CO}-\mathrm{N}_{2}$ qui caractérise la transformation quasi instantanée de l'oxygène pur (qui n'apparaît pas sur le spectre) sous la forme de CO. Le carbone provenant de l'enceinte où il a pu être introduit en particulier avec un échantillon nettoyé à l'alcool éthylique. La figure 4 représente l'évolution des pressions partielles dans l'enceinte pendant l'oxydation.

De la même manière que ci-dessus, nous avons oxydé la surface et relevé les spectres Auger, la figure 5

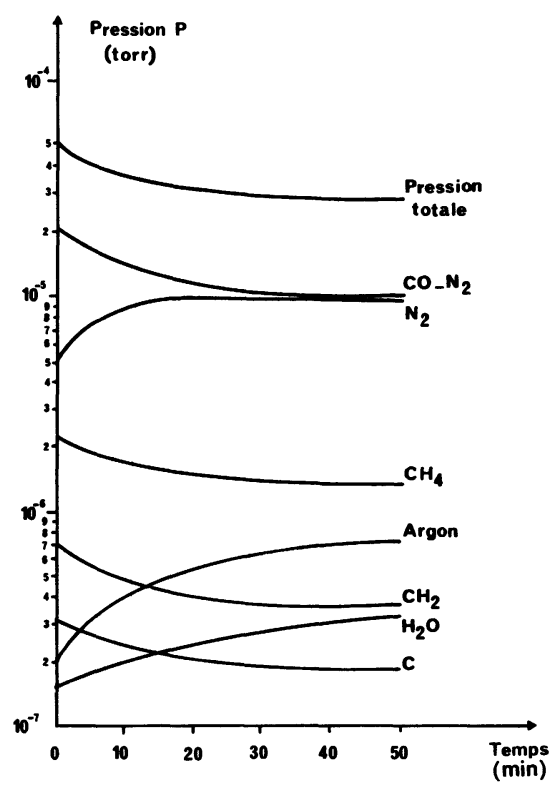

Fig. 4. - Evolution des pressions partielles dans l'enceinte pendant l'oxydation par le faisceau électronique après introduction d'oxygène.

[Partial pressures evolution in the vacuum device during the oxidation by the electron beam after oxygen introduction.]

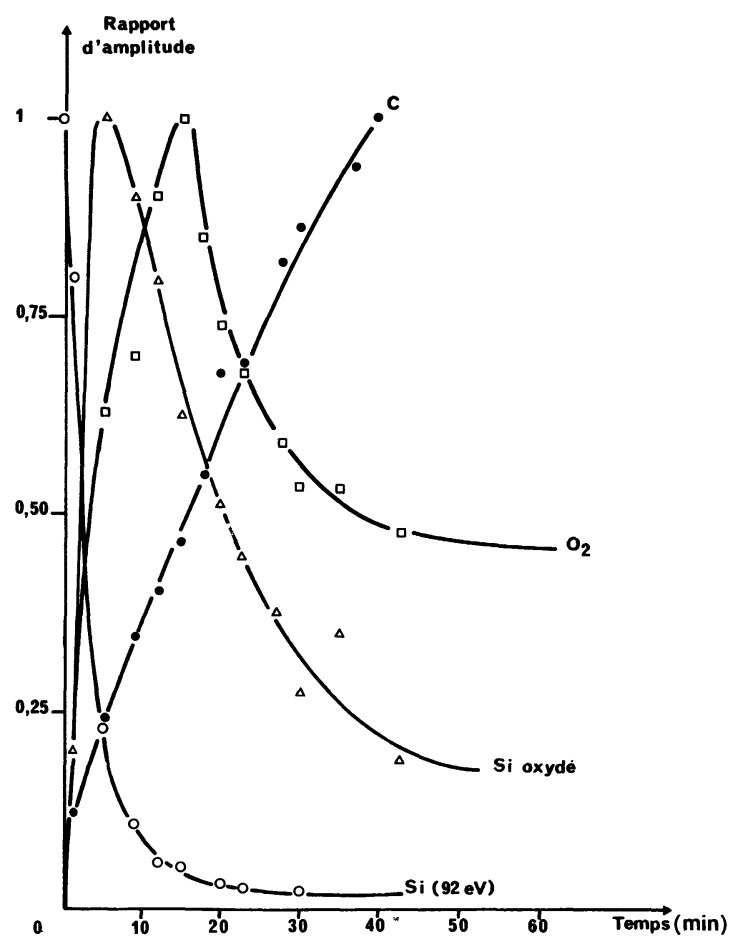

Fig. 5. - Evolution de l'amplitude des pics Auger pendant l'oxydation du silicium sous oxygène sec en régime statique et pour une énergie du faisceau électronique de $1 \mathrm{keV}$.

[Evolution of Auger peaks amplitude during silicon oxidation under dry oxygen in static regime and for an electron beam energy of $1 \mathrm{keV}$.] 
représente l'évolution des pics Auger. Au début on a une oxydation très rapide sous le faisceau électronique; après quelques minutes de bombardement électronique on obtient un spectre d'oxyde comparable à celui obtenu en fin d'oxydation sans entrée d'oxygène mais avec un pic de carbone plus important. Ensuite le pic de carbone continue à croître puis l'oxyde préalablement formé est recouvert d'une couche de carbone, d'où une décroissance de l'amplitude des pics relatifs au silicium oxydé et à l'oxygène.

3.3 OXYDATION PAR BOMBARDEMENT ÉLECTRONIQUE SOUS FLUX D'OXYGÈNE SEC. - Afin d'éviter la transformation de l'oxygène dans l'enceinte sous forme de $\mathrm{CO}$, nous avons choisi d'oxyder notre échantillon sous un flux constant d'oxygène. La vanne d'introduction est ouverte, et le débit de la fuite de $\mathrm{O}_{2}$ réglé de manière à obtenir une pression totale dans l'enceinte de 2,5 $\times 10^{-7}$ torr, la vanne de pompage étant partiellement fermée. La pression partielle d'oxygène est de $3 \times 10^{-8}$ torr.

L'échantillon étudié a subi le même traitement de nettoyage que le précédent. Nous suivons l'évolution de l'oxydation sous le faisceau au cours du temps (Fig. 6). L'oxydation est très rapide et se caractérise par la diminution de l'amplitude du pic silicium pur Si $(92 \mathrm{eV})$ et la croissance des pics silicium oxydé $(\mathrm{Si} 76 \mathrm{eV})$ et oxygène $(507 \mathrm{eV})$. Les différents stades de l'oxydation décrits lors de l'oxydation sous atmosphère résiduelle sont visibles et ne présentent pas de différences évidentes. Les pics Si oxydé et oxygène tendent rapidement vers un maximum. La différence essentielle concerne le pic carbone. Celui-ci apparaît lors des premiers spectres d'oxydation c'est-à-dire

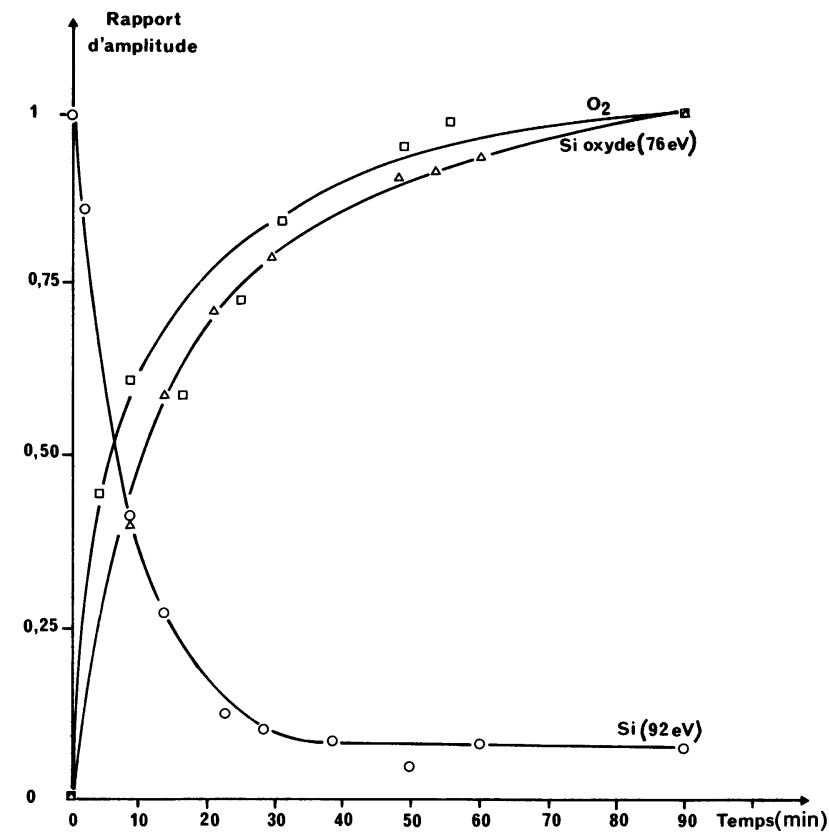

Fig. 6. - Evolution de l'amplitude des pics Auger au cours de l'oxydation par bombardement électronique sous flux d'oxygène constant.

[Evolution of Auger peaks amplitude during silicon oxidation by an electron beam under constant oxygen flow.]

après ouverture de la fuite d'oxygène. Son amplitude diminue au cours du temps pour disparaître complètement après environ une heure de bombardement électronique, il est entièrement noyé dans le bruit de fond (Fig. 7). Cette disparition coïncide avec la

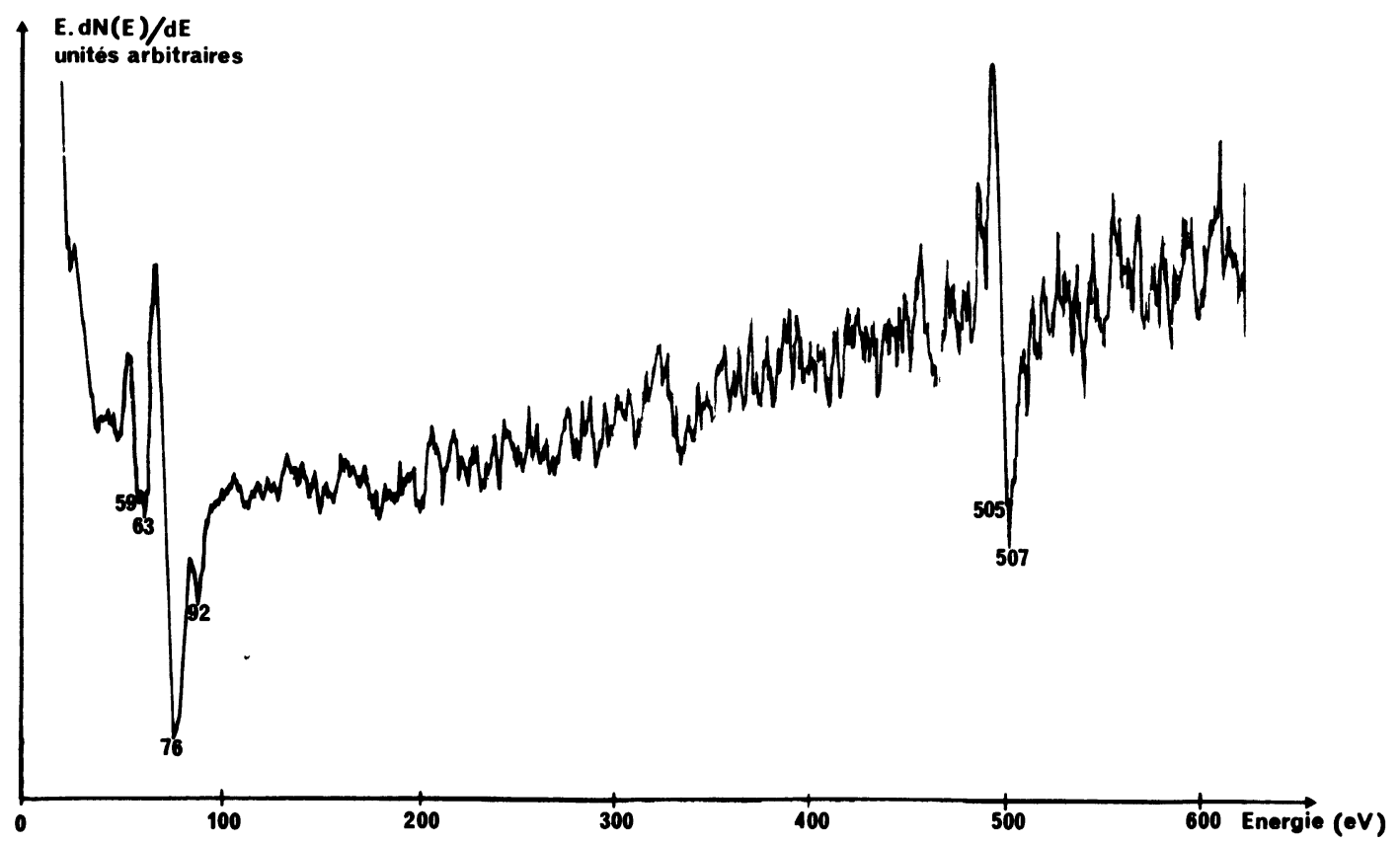

Fig. 7. - Spectre Auger de l'oxyde obtenu après oxydation par bombardement électronique sous flux d'oxygène sec.

[Auger spectrum obtained after oxidation by an electron beam under dry oxygen flow.] 
translation des pics du silicium oxydé qui passent respectivement de 63,68 et $80 \mathrm{eV}$ à 59,63 et $76 \mathrm{eV}$ correspondant aux énergies relevées sur de l'oxyde pur. La transition des pics s'effectue par une réduction de la largeur des pics enregistrés qui deviennent plus pointus. Le pic oxygène étant lui à $507 \mathrm{eV}$, énergie de l'oxygène dans le $\mathrm{SiO}_{2}$.

3.4 COMPARAISON DES DIFFÉRENTS OXYDES OBTENUS ENTRE EUX ET AUX OXYDES THERMIQUES. - Les études effectuées montrent une dépendance très importante entre l'oxydation et les gaz résiduels dans l'enceinte. L'étude de l'oxydation sous oxygène statique fait apparaître quatre stades visibles en spectrométrie Auger. Ce phénomène apparaît également lors de l'oxydation après fermeture de la vanne de pompage et après introduction d'un gaz neutre dans l'enceinte. Ces différents stades se caractérisent par une modification des spectres du silicium pur (Fig. 2) :

- une déformation du sommet du pic à $92 \mathrm{eV}$,

- l'apparition d'un pic intermédiaire à $83-84 \mathrm{eV}$ révélant la formation d'un oxyde de composition indéfinie souvent appelé $\mathrm{SiO}_{x}$,

- la croissance du pic de l'oxyde $\mathrm{SiO}_{2}$ (76 ou $80 \mathrm{eV}$ ) avec la disparition du pic intermédiaire,

- la saturation de la couche d'oxyde de silicium caractérisée par l'amplitude maximum du pic de l'oxyde.

Ces résultats sont en accord avec ceux obtenus par différents auteurs $[7,8]$ et en particulier avec la publication de Carrière et al. [3] qui ont également étudié les premiers stades de l'oxydation à température ambiante et sous une faible pression d'oxygène $\left(5 \times 10^{-6}\right.$ torr). Derrein et Commandré [5] et Rigo [6] ont aussi étudié les premières phases de l'oxydation thermique du silicium et ils proposent une cinétique de croissance avec diffusion de l'oxygène au travers de l'oxyde.

L'oxydation sous flux d'oxygène se démarque des deux premières études par le fait que le pic carbone n'apparaît pas sur l'oxyde obtenu après un certain temps d'oxydation et que l'énergie des pics du silicium oxydé sont alors à 59,63 et $76 \mathrm{eV}$ et celui de l'oxygène à $507 \mathrm{eV}$ comme pour l'oxyde thermique après nettoyage ionique [1].

Dans le cas des oxydations effectuées ci-dessus sous atmosphère résiduelle et sous oxygène statique, il y aurait formation d'une couche superficielle de stœchiométrie $\mathrm{SiO}_{x} \mathrm{C}_{y}$ sous l'influence du faisceau d'électrons dans une atmosphère comportant des composés carbonés. Ce qui modifierait les niveaux d'énergie électronique et provoquerait une translation de $4 \mathrm{eV}$ de l'énergie des pics du silicium oxydé. Dans le cas de l'oxydation sous flux d'oxygène ce composé serait obtenu au début de l'oxydation et ensuite on obtiendrait l'oxyde de silicium $\mathrm{SiO}_{2}$ comme dans le cas de l'oxyde thermique.

\section{Conclusion.}

Les résultats obtenus montrent qu'il est possible d'oxyder la surface de silicium par bombardement électronique sous de très faibles pressions d'oxygène, mais les résultats obtenus sont très sensibles aux conditions expérimentales. La présence de composés à base de carbone dans l'enceinte à vide a une influence sur la composition de la couche d'oxyde réalisée; ces composés peuvent, par exemple, être introduits par un échantillon qui vient d'être nettoyé à l'alcool éthylique. La transition entre le substrat de silicium et la couche d'oxyde est très dépendante des conditions d'oxydation et nous avons précisé les différentes étapes de l'oxydation.

Il nous semble intéressant de procéder à une étude similaire de l'oxydation thermique non seulement sous faible pression d'oxygène mais également sous des pressions proches de (ou égale à) la pression atmosphérique et de voir l'impact sur les propriétés des dispositifs électroniques réalisés avec ces oxydes.

\section{Bibliographie}

[1] Chartier, J. L., Le Bihan, R., Pilorget, L., Revue Phys. Appl. 19 (1984) 927-931.

[2] Joyce, B. A. and Neave, J. H., Surface Sci. 34 (1973) 401-419.

[3] Carrière, B., Chouiyakh, A. and Lang, B., Surface Sci. 126 (1983) 495-501.

[4] Legare, P., Maire, G., Carrière, B. and Deville, J. P., Surface Sci. 68 (1967) 348-358.

[5] Derrien, J. and Commandre, M., Surface Sci. 118 (1982) 32-46.

[6] Rigo, S., Entretiens de Toulouse sur la microélectronique (GCIS), 15-16 décembre 1983.
[7] a. Munoz, M. C., Sacedon, J. L., J. Chem. Phys. 74 (8) (1981) 4693-4700.

b. Munoz, M. C., Martinez, V., Tagle, J. A., SaceDON, J. L., J. Phys. C : Solid State Physics 13 (1980) 4247-62.

c. Munoz, M. C. et al., Phys. Rev. Lett. 44 (12) (1980) 814-17.

[8] a. Lang, B., Mosser, A., J. Microsc. Spectrosc. Electron. 6 (1981) 131-140.

b. Lang, B., Scholler, P., Carrière, B., Surface Sci. 99 (1980) 103. 